\title{
Caracterização assistencial dos hospitais filantrópicos da Região Metropolitana de Belo Horizonte
}

\author{
Assistance characterization of philanthropic hospitals of \\ the Metropolitan Area of Belo Horizonte
}

\author{
Daniel Penido de Lima Amorim ${ }^{1}$ (D), Antônio Artur de Souza ${ }^{1}$
}

\begin{abstract}
Resumo
Introdução: Os hospitais filantrópicos compreenderem cerca de um terço do parque hospitalar brasileiro, apresentando grande participação nas internações para o SUS. Muitos deles são estratégicos devido a se tratar das únicas unidades hospitalares em seus municípios. Objetivo: Este estudo objetivou analisar a distribuição geográfica, a complexidade e a capacidade assistencial dos hospitais filantrópicos da Região Metropolitana de Belo Horizonte (RMBH), inclusive, comparando com tendências encontradas no âmbito nacional. Adicionalmente, quando o município apresentou apenas um hospital, sendo ele filantrópico, buscou-se verificar se a capacidade assistencial atingia o mínimo recomendado pela OMS. Método: Todos os dados empregados foram coletados no DATASUS. Utilizou-se de dados de estabelecimentos para descrever a distribuição geográfica e a complexidade assistencial. A análise da capacidade assistencial exigiu o cálculo de Leitos de Hospitais Filantrópicos por Mil Habitantes $\left(\mathrm{L}_{\mathrm{HF}} /{ }_{\mathrm{M}} \mathrm{H}\right)$, utilizando-se de dados de recursos físicos e população. Resultados: $\mathrm{Na} \mathrm{RMBH}$, os hospitais filantrópicos gerais, em sua maioria, estão distribuídos individualmente pelos municípios menores, enquanto os especializados concentram-se na capital. $O \mathrm{~L}_{\mathrm{HF}} /{ }_{\mathrm{M}} \mathrm{H}$ cresceu na RMBH, na capital e no conjunto dos municípios menores. O único município, dentre aqueles que possuem apenas um hospital, sendo ele filantrópico, que apresentou $\mathrm{L}_{\mathrm{HF}}{ }_{\mathrm{M}} \mathrm{H}$ maior que o mínimo recomendado pela OMS foi Itaguara. Conclusão: Percebeu-se que há grande semelhança entre os recortes nacional e aquele da RMBH. Muitos municípios dessa região apresentam apenas uma unidade hospitalar, sendo ela filantrópica, fato que corrobora a importância dessas organizações para a saúde local.

Palavras-chave: hospital filantrópico; Região Metropolitana de Belo Horizonte; capacidade assistencial; saúde pública; saúde coletiva.

Abstract

Background: The philanthropic hospitals comprise about one-third of the Brazilian hospital sector, with large participation in hospitalizations for the SUS. Many of them are strategic because they are the only hospital units in their municipalities. Objective: This study aimed to analyze the geographic distribution, the healthcare complexity and the hospital bed capacity of the philanthropic hospitals of the Metropolitan Area of Belo Horizonte (RMBH), comparing with tendencies found in the national scope. Additionally, when the municipality presented only one hospital, being philanthropic, it was sought to verify if the hospital bed capacity reaches the minimum recommended by the WHO. Method: All data used were collected in DATASUS. Data from establishments were used to describe the geographic distribution and healthcare complexity. The analysis of hospital bed capacity required the calculation of Philanthropic Hospital Beds per Thousand Inhabitants $\left(\mathrm{L}_{\mathrm{HF}} / \mathrm{M}\right)$, using data from physical resources and population. Results: In the $\mathrm{RMBH}$, the general philanthropic hospitals, in the majority, are distributed individually by the smaller municipalities, whereas the specialized ones are concentrated in the capital. $\mathrm{L}_{\mathrm{HF}} / \mathrm{M}$ H grew in $\mathrm{RMBH}$, in the capital and in the set of smaller municipalities.
\end{abstract}

${ }^{1}$ Centro de Pós-graduação e Pesquisa em Administração (CEPEAD), Universidade Federal de Minas Gerais (UFMG) - Belo Horizonte (MG), Brasil.

Trabalho realizado na Universidade Federal de Minas Gerais (UFMG) - Belo Horizonte (MG), Brasil.

Endereço para correspondência: Daniel Penido de Lima Amorim - Centro de Pós-graduação e Pesquisa em Administração (CEPEAD), Universidade Federal de Minas Gerais (UFMG), Avenida Presidente Antônio Carlos, 6627, Sala 4012 - Pampulha - CEP: 31270-901 - Belo Horizonte (MG), Brasil -

Email: daniel_amorim23@hotmail.com

Fonte de financiamento: nenhuma.

Conflito de interesses: nada a declarar.

Este é um artigo publicado em acesso aberto (Open Access) sob a licença Creative Commons Attribution, que permite uso, distribuição e reprodução em qualquer meio, sem restrições desde que o trabalho original seja corretamente citado. 
Itaguara was the only municipality, among those who only have one hospital, being philanthropic, which presented $\mathrm{L}_{\mathrm{HF}} /{ }_{\mathrm{M}} \mathrm{H}$ higher than the minimum recommended by WHO. Conclusion: There is great similarity between the national cuts and the RMBH. Many municipalities in this region have only one hospital unit, being philanthropic, a fact that corroborates the importance of these organizations for local health.

Keywords: charity hospitals; Metropolitan Area of Belo Horizonte; hospital bed capacity; public health; collective health.

\section{INTRODUÇÃO}

Os hospitais são as principais organizações, dentre aquelas que compreendem o diversificado sistema de saúde brasileiro, os quais apresentam como função, basicamente, ofertar assistência médica integral, curativa e preventiva à população ${ }^{1}$. Eles podem ser divididos entre públicos, filantrópicos e com fins lucrativos.

O foco deste estudo são os hospitais filantrópicos, os quais ofertam aos usuários não apenas serviços privados, como também serviços de natureza social com propósitos beneficentes, possibilitados, principalmente, por meio de associações com o Sistema Único de Saúde (SUS) ${ }^{2}$. Esses hospitais são dignos de atenção por compreenderem cerca de um terço do parque hospitalar brasileiro, apresentando também grande participação nas internações para o SUS. Além disso, muitos deles são considerados estratégicos para a atenção à saúde de suas regiões, tratando-se, muitas vezes, das únicas unidades hospitalares em seus municípios ${ }^{3-5}$.

Apesar dessa importância, no Brasil poucos estudos trataram das características assistenciais dos hospitais filantrópicos. Alguns deles mostraram que, no âmbito nacional, os hospitais especializados tendem a estar concentrados nas capitais, enquanto os hospitais gerais, em sua maioria, são distribuídos pelos municípios do interior ${ }^{3,5}$. Assim, existe uma relação entre a distribuição geográfica e a complexidade assistencial desses hospitais. Entretanto, não foram compartilhadas informações regionais e, inclusive, não são conhecidos estudos sobre os hospitais filantrópicos da Região Metropolitana de Belo Horizonte (RMBH).

Nesse sentido, também não se conheciam estudos que buscassem avaliar a capacidade assistencial dos hospitais filantrópicos dessa região. Essa capacidade é, geralmente, mensurada pelo indicador Leitos por Mil Habitantes $\left(\mathrm{L} /{ }_{M} \mathrm{H}\right), \mathrm{o}$ qual relaciona os leitos existentes em hospitais de uma localidade com a demanda da população nela residente. Sabe-se, porém, que desde o final dos anos 1990 o sistema de saúde brasileiro, visto como um todo, não apresentava a capacidade assistencial mínima de três $L /{ }_{M} H$ recomendada pela Organização Mundial da Saúde (OMS) ${ }^{6}$.

Tendo em vista que informações regionais são essenciais para o planejamento, gestão e avaliação dos serviços de saúde ${ }^{7}$, este estudo objetivou levantar a distribuição geográfica dos hospitais filantrópicos da RMBH, bem como analisar a complexidade e a capacidade assistencial dessas organizações. Buscou-se também fazer um paralelo entre tendências verificadas no âmbito nacional e aquelas inferidas na RMBH. Adicionalmente, nos casos em que os municípios apresentaram apenas um hospital, sendo ele filantrópico, foi possível verificar se a capacidade assistencial atingiu o mínimo recomendado pela OMS. Assim, foram geradas informações úteis aos gestores das organizações públicas e privadas do setor de saúde.

\section{Caracterização dos hospitais filantrópicos no âmbito nacional}

Tradicionalmente, os hospitais filantrópicos são aqueles que ofertam “[...] serviços de natureza social com propósitos beneficentes aos seus usuários, seja através de associações típicas com o Estado, como é o caso do SUS, seja isoladamente"2 (p. 1). Todavia, legalmente, o que define um hospital como filantrópico é dispor do Certificado de Entidade Beneficente de Assistência Social regularizado ${ }^{2,4,5}$.

Diversos estudos sobre esses hospitais foram realizados pelos pesquisadores vinculados à Escola Nacional de Saúde Pública da Fundação Oswaldo Cruz. Dentre eles, pode-se destacar o de Barbosa et al. ${ }^{3}$ e o de Portela et al. ${ }^{5}$, que caracterizaram essas organizações no âmbito nacional. Conforme os resultados neles encontrados, esses hospitais apresentam uma rede espalhada desde as capitais até os menores municípios interioranos, contemplando cerca de um terço dos leitos existentes no país. Pode-se, ainda, perceber que existe uma relação entre a localização geográfica e o nível de complexidade assistencial desses estabelecimentos.

Existem dois grupos distintos de hospitais filantrópicos. O primeiro é composto por um vasto número de hospitais de baixa complexidade, os gerais, que tendem a ser providos de um pequeno número de leitos e a estar localizados nos municípios do interior. Frequentemente, esses hospitais são a única unidade hospitalar de seus municípios ${ }^{3,5,8}$, tornando-se estratégicos para o atendimento de pacientes mais vulneráveis. Entretanto, sofrem dificuldades em relação à falta de equipamentos médico-hospitalares e à retenção de profissionais ${ }^{5,9}$.

Por outro lado, o segundo grupo é composto por poucos hospitais de alta complexidade, os especializados, que tendem a ser providos de grande número de leitos e a localizar-se em cidades maiores, principalmente em capitais. Esse grupo compreende, inclusive, alguns hospitais com elevada capacidade tecnológica, os quais realizam procedimentos de alta complexidade e possuem reconhecimento internacional no tratamento de determinadas doenças ${ }^{3,5,8}$. 


\section{Indicador de capacidade de assistencial}

O indicador chamado Leitos por Mil Habitantes $\left(L /{ }_{M} H\right)$ é o principal dentre aqueles utilizados para avaliar a capacidade assistencial em localidades, conceituado pela Rede Interagencial de Informação para a Saúde (RIPSA) ${ }^{10}$ (p. 240) como "[...] número de leitos hospitalares públicos e privados, vinculados ou não ao Sistema Único de Saúde (SUS), por mil habitantes, em determinado espaço geográfico, no ano considerado". Nesse sentido, além de leitos e população, leva em consideração uma determinada localidade e um momento no tempo.

Normalmente, esse indicador é utilizado para avaliar a disponibilidade de leitos hospitalares públicos e privados - ou públicos, privados e filantrópicos, caso segregado os filantrópicos, os quais são privados, ainda que sem fins lucrativos -, como proporção da população residente em uma determinada região. Nesse sentido, leva em conta tanto a capacidade de atendimento dos hospitais, quanto a demanda da população por serviços de saúde $^{10}$. O mesmo permite verificar situações de desequilíbrio na distribuição de leitos entre regiões ${ }^{10,11}$. Análises decorrentes de sua aplicação podem prover informações importantes aos processos de planejamento, gestão e avaliação de políticas voltadas para a assistência médico-hospitalar ${ }^{10,12,13}$.

$\mathrm{O}$ indicador $L /{ }_{M} H$ é influenciado por fatores socioeconômicos, epidemiológicos e demográficos, tais como nível de renda, composição etária, oferta de profissionais de saúde, políticas públicas assistenciais e preventivas ${ }^{10,12,13}$. "Em geral, a concentração de leitos está associada ao maior poder aquisitivo da população e à demanda por serviços especializados, condições que atraem investimentos do setor privado de saúde"10 (p. 240).

Rodrigues ${ }^{11}$, utilizando-se desse indicador, relatou que na década de 1980 predominava uma grande disparidade nos serviços de saúde, tanto nas regiões intraestaduais, quanto nas unidades federativas do Brasil. Segundo ele, na época, a disponibilidade de leitos era condição suficiente para sua ocupação. A situação nos anos 2000 não parecia ser muito diferente, visto que Castro et al. ${ }^{14}$ encontraram uma forte relação entre a oferta de serviços de saúde e o uso das internações hospitalares, sugerindo um efeito de demanda induzida pela oferta de leitos.

As limitações do indicador $L /{ }_{M} H$ são: os leitos podem ser ocupados por não residentes, distorcendo a disponibilidade de serviços para a população do município; ausências de padrões validados para comparações, uma vez que o indicador expressa uma combinação de fatores inerentes a diferentes realidades regionais ou locais ${ }^{10}$; e necessidade de ser usado em associação com informações como perfil epidemiológico da população, organização dos serviços de saúde entre vários tipos de estabelecimentos, disponibilidade de recursos tecnológicos e existência de serviços especializados ${ }^{15}$.

Nesse sentido, Rodrigues ${ }^{11}$ adotou esse indicador junto a outros relacionados à mortalidade, internações e despesas, em sua análise sobre a distribuição dos recursos de saúde no Brasil. De outro modo, Luiz et al. ${ }^{16}$ utilizaram-se desse indicador e de outras variáveis para construir um índice que permitisse o reconhecimento das desigualdades de condições de vida e saúde da população de diferentes regiões brasileiras.

Segundo dados do Instituto Brasileiro de Geografia e Estatística (IBGE) $)^{6}$, o indicador $L /{ }_{M} H$, que inclui leitos de hospitais públicos, privados e filantrópicos, caiu continuamente entre 1992 e 2009, tanto para o Brasil quanto para o estado de Minas Gerais e para a Região Metropolitana de Belo Horizonte. O Ministério da Saúde ${ }^{17}$ divulgou justificativas para essa queda, a saber: políticas preventivas, redução do número de nascimentos e redução das necessidades de internação devido aos avanços tecnológicos nos tratamentos. Anderson et $\mathrm{al}^{18}$. relatam que essa redução ocorreu na maioria dos países-membros da Organização para a Cooperação e Desenvolvimento Econômico (OCDE) ao longo da década de 1990.

\section{MÉTODO}

Os dados utilizados nas análises deste estudo descritivo são originários do Cadastro Nacional de Estabelecimentos de Saúde (CNES) e do IBGE. Eles foram coletados no banco de dados chamado Departamento de Informática do Sistema Único de Saúde (DATASUS), que é de acesso livre via internet. O período de análise selecionado foi de 2006 a 2014, e os dados de estabelecimentos de saúde e leitos hospitalares correspondem ao mês de janeiro. Esse mês foi escolhido aleatoriamente, visto que quantidades de leitos e de hospitais variam pouco mensalmente.

No DATASUS foi gerado um recorte de acordo com: tipos de estabelecimentos, tipos de prestador - filantrópico, público ou privado -, e municípios aos quais os hospitais pertencem. A análise concentrou-se nos dados de hospitais filantrópicos, uma vez que essas organizações foram o foco do estudo.

Primeiramente, analisou-se a distribuição geográfica, a complexidade assistencial e evolução do número de hospitais. Para isso, foram selecionados quatro tipos de estabelecimentos de saúde presentes no CNES, a saber: Hospital Geral, Hospital Especializado, Pronto-Socorro Geral e Pronto-Socorro Especializado.

Para a análise da capacidade assistencial dos hospitais foram selecionados dois tipos dentre os recursos físicos presentes no CNES: Leitos de Internação e Leitos Complementares. Essa análise se deu por meio do cálculo e avaliação do indicador Leitos em Hospitais Filantrópicos por Mil Habitantes (doravante, $L_{H F} /{ }_{M} H$ ), uma adaptação do $L /{ }_{M} H$ desenvolvida para não englobar os leitos de hospitais públicos e com fins lucrativos. Seu cálculo é simples: o número de leitos presentes nos hospitais filantrópicos de uma localidade é multiplicado por mil e dividido pelo número de habitantes dessa localidade. 
Porém, dois métodos de cálculo distintos foram empregados, conforme as diferentes localidades que se pretendeu analisar. O Método I considerou o número de habitantes do conjunto de municípios, independentemente de todos apresentarem ou não hospitais filantrópicos. Por outro lado, o Método II considerou apenas o número de habitantes dos municípios que apresentam hospitais filantrópicos. As Fórmulas 1 e 2 desses métodos são apresentadas a seguir.

Método $\mathrm{I}: \mathrm{L}_{\mathrm{HF}} / \mathrm{mH}=\frac{\mathrm{L}_{\text {todos os municípios }} \times 1.000}{\mathrm{H}_{\text {todos os municípios }}}$

Método II : $\mathrm{L}_{\mathrm{HF}} / \mathrm{mH}=\frac{\mathrm{L}_{\text {municípios com } \mathrm{HF}} \times 1.000}{\mathrm{H}_{\text {municípios com } \mathrm{HF}}}$

Nas fórmulas, $L$ representa o número de leitos e $H$ o número de habitantes. HF é meramente a abreviação de hospitais filantrópicos.

Entendeu-se que a demanda por leitos na $\mathrm{RMBH}$ deveria levar em conta toda a população dessa região, por conseguinte, no seu cálculo de $L_{H F}{ }_{M} H$ adotou-se o Método I. Por outro lado, para Belo Horizonte e o conjunto de municípios menores adotou-se o Método II.

$\mathrm{Na}$ análise, os municípios semelhantes aos que Portela et al. ${ }^{5}$ se referiam como do interior serão tratados como municípios menores, pois se tratando de uma região metropolitana, nem todos terão, de fato, características de interior, ainda que sejam menores que Belo Horizonte.

\section{RESULTADOS E DISCUSSÃO}

Distribuição geográfica e caracterização assistencial dos hospitais filantrópicos

A RMBH é constituída por 34 municípios, a saber: Baldim, Belo Horizonte, Betim, Brumadinho, Caeté, Capim Branco, Confins, Contagem, Esmeraldas, Florestal, Ibirité, Igarapé, Itaguara, Itatiaiuçu, Jaboticatubas, Juatuba, Lagoa Santa, Mário Campos, Mateus Leme, Matozinhos, Nova Lima, Nova União, Pedro Leopoldo, Raposos, Ribeirão das Neves, Rio Acima, Rio Manso, Sabará, Santa Luzia, São Joaquim de Bicas, São José da Lapa, Sarzedo, Taquaraçu de Minas e Vespaziano.

O conjunto total dos hospitais da $\mathrm{RMBH}$, incluindo hospital geral, hospital especializado, pronto-socorro geral e pronto-socorro especializado, está distribuído na seguinte proporção: 53\% de hospitais privados, $25 \%$ de hospitais públicos e $22 \%$ de hospitais filantrópicos. Apesar de os hospitais filantrópicos serem minoria, eles são importantes porque, em muitos casos, eles consistem na única unidade hospitalar em municípios do interior. As análises seguintes tratarão dos hospitais filantrópicos gerais e especializados, pois não existiam hospitais filantrópicos do tipo pronto-socorro na RMBH durante o período analisado.

Dentre os 34 municípios pertencentes a essa região, apenas 11 apresentam hospitais filantrópicos. A Figura 1 é um mapa da $\mathrm{RMBH}$, no qual os municípios que apresentam hospitais filantrópicos estão destacados em tom cinza.

Pela análise dos dados referentes ao número de hospitais filantrópicos presentes na RMBH, percebeu-se que entre 2006 e 2014 a quantidade desses hospitais manteve-se praticamente

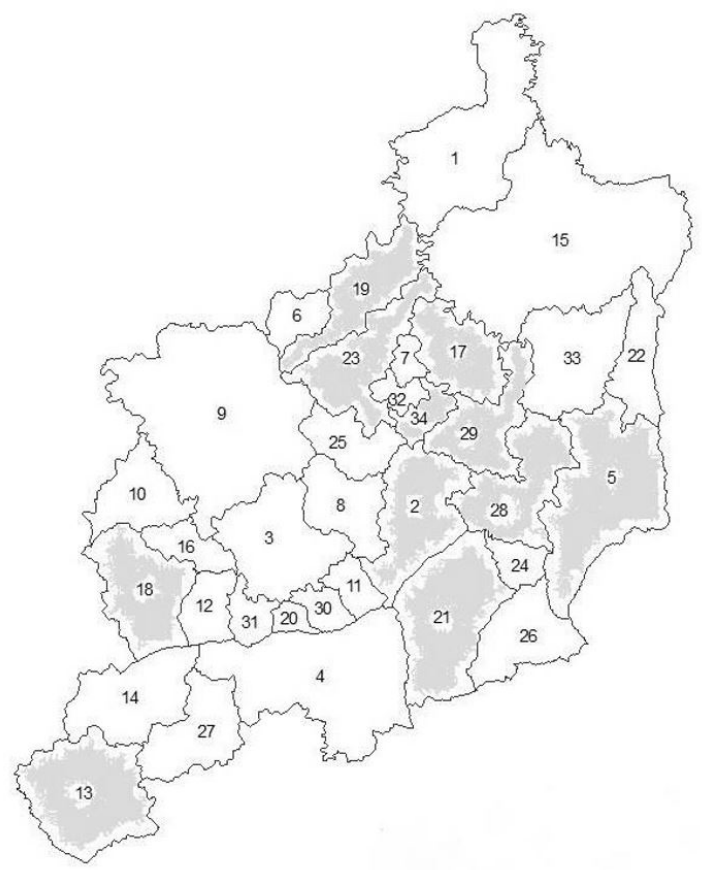

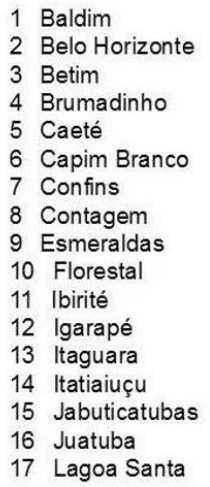
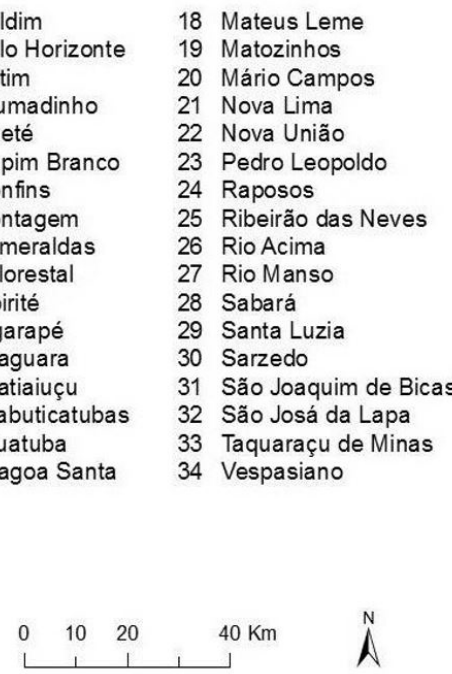

Figura 1. Municípios que apresentam Hospitais Filantrópicos na Região Metropolitana de Belo Horizonte. Os municípios que possuem hospitais filantrópicos estão destacados em tom cinza 
estável. Foi encontrada uma série de semelhanças entre o recorte no âmbito nacional, que foi descrito por Barbosa et al. ${ }^{3}$ e por Portela et al. ${ }^{5}$, e aquele no âmbito da RMBH, a começar pelo número de hospitais gerais ser representativo da maioria dos hospitais filantrópicos. Enquanto havia 19 hospitais gerais em 2014, os hospitais especializados eram apenas quatro. Ou seja, a proporção era de $83 \%$ de hospitais gerais para $17 \%$ de especializados nesse ano.

A distribuição dos hospitais filantrópicos pelos municípios também remete à lógica que prevalece no âmbito nacional. Os municípios menores, quando apresentam hospitais filantrópicos, têm seu número limitado a apenas uma unidade. Dentre eles, $60 \%$ têm esse estabelecimento como a única unidade hospitalar, independentemente de sua classificação quanto ao tipo de prestador - público, privado ou filantrópico. São eles: Caeté, Itaguara, Mateus Leme, Matozinhos, Santa Luzia e Vespasiano.

Os municípios de Lagoa Santa, Sabará e Pedro Leopoldo apresentam um hospital filantrópico e um privado, cada. Nova Lima apresenta dois hospitais privados e um filantrópico. O único município que conta com mais de um hospital filantrópico é a capital, Belo Horizonte, apresentando 13 hospitais, correspondentes a 53\% daqueles presentes na RMBH.

De modo semelhante à tendência mostrada por Barbosa et al. ${ }^{3}$ e Portela et al. ${ }^{5}$, na RMBH os municípios menores apresentam somente hospitais gerais, enquanto a capital concentra os hospitais especializados. Em 2014, Belo Horizonte apresentava quatro hospitais filantrópicos especializados e nove gerais, isto é, aproximadamente $30 \%$ de especializados e $70 \%$ de gerais. Com isso, percebe-se que, apesar da propensão dos hospitais filantrópicos gerais se localizarem em municípios menores, mesmo na capital seu número era mais que o dobro daquele de hospitais especializados.

\section{Leitos de hospitais filantrópicos por mil habitantes}

A relação entre o total de leitos hospitalares e a população das localidades é de grande importância para os órgãos governamentais avaliarem se há a necessidade de expansão dos serviços públicos de saúde. Essa informação também é útil para estudos de viabilidade para a instalação de hospitais privados, os quais consideram a demanda por serviços de saúde na região.

No entanto, deve-se ter em mente que os dados mostrados a seguir são referentes apenas aos leitos de hospitais filantrópicos, excluindo a parcela de capacidade assistencial dos hospitais públicos e privados. Por isso, não devem ser usados para avaliar a capacidade total de atendimento hospitalar nas localidades que também apresentam hospitais públicos e com fins lucrativos.

Segundo os dados do IBGE $\mathrm{IB}^{6}$ o número de $L /{ }_{M} H$, referente ao conjunto de hospitais públicos, privados e filantrópicos, caiu continuamente na RMBH nos anos 2000. Porém, o mesmo não acontece quando observado o indicador que considera apenas os leitos dos hospitais filantrópicos. Ao contrário, o $L_{H F} /{ }_{M} H$ seguiu uma tendência de crescimento ao longo desses anos. Os dados mostrados no Gráfico 1 indicam que esse indicador cresceu tanto no total da RMBH quanto na capital e nos municípios menores.

Esse gráfico mostra que o $L_{H F} /{ }_{M} H$ na RMBH passou de 0,43 , em 2006, para 0,60, em 2014, o equivalente a um crescimento de $41 \%$. Cabe lembrar que o Método $I$, que foi aplicado no cálculo do indicador para essa região, leva em conta a população dos municípios que não apresentam hospitais filantrópicos. Isso explica por que os valores para a RMBH foram inferiores aos da capital e do grupo dos municípios menores.

$\mathrm{O} L_{H F} /{ }_{M} H$ calculado para o conjunto dos municípios menores e para a capital, que adota o Método II, revela que ambas localidades apresentaram valores próximos desse indicador. Na capital, ele passou de 0,65, em 2006, para 0,99, em 2014, o equivalente a um crescimento de $52 \%$. No conjunto dos municípios menores, passou de 0,75, em 2006, para 0,82, em 2014, o equivalente a um crescimento de $9 \%$. As médias dos indicadores anuais, no período de 2006 até 2014, para o conjunto dos municípios menores e para a capital foram 0,85 e 0,87 , respectivamente. Ou seja, os dois recortes apresentaram o $L_{H F}{ }_{M} H$ bem próximo, se considerado todo o período.

A Tabela 1 mostra o $L_{H F} /{ }_{M} H$ em cada município pertencente à RMBH que apresenta o tipo de organização em questão. O pequeno município de Itaguara se destaca como aquele que possui o maior $L_{H F}{ }_{M} H$, enquanto o município de Sabará é aquele que possui o menor.

Como já mencionado, os municípios que apresentam apenas um hospital, sendo ele filantrópico, são: Caeté, Itaguara, Mateus Leme, Matozinhos, Santa Luzia e Vespasiano. Devido a esse hospital ser o único do município, seu $L_{H F} /{ }_{M} H$ engloba a totalidade leitos presentes na localidade, portanto, nesse caso o $L_{H F} /{ }_{M} H$ é igual ao $L /{ }_{M} H$. Por isso, esse indicador pode ser comparado com o mínimo de três $L /{ }_{M} H$ indicado pela a OMS. Assim, dentre esses municípios, o único que atinge esse número mínimo é Itaguara.

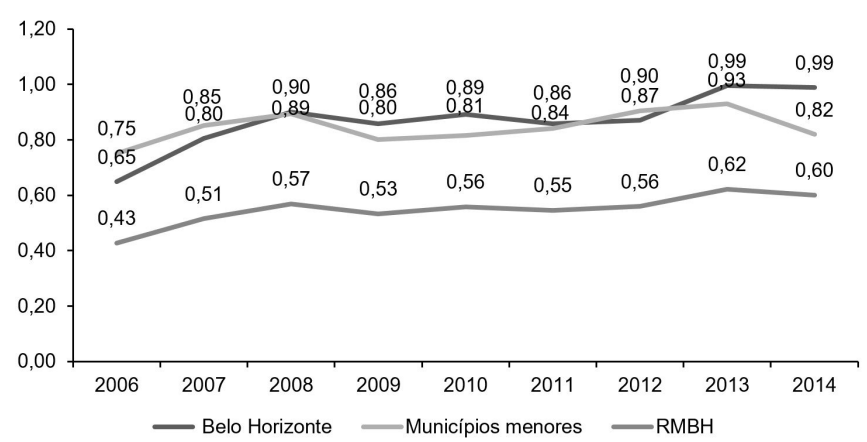

Gráfico 1. Leitos de Hospitais Filantrópicos por Mil Habitantes na Região Metropolitana de Belo Horizonte (2006-2014) 
Tabela 1. Leitos de Hospitais Filantrópicos por Mil Habitantes em Municípios da Região Metropolitana de Belo Horizonte (2014)

\begin{tabular}{lcccc}
\multicolumn{1}{c}{ Município } & Leitos $_{\mathrm{HF}}$ & \% de $\mathbf{L}_{\mathbf{H F}}$ no total & Habitantes & $\boldsymbol{L}_{\boldsymbol{H F}} \mathbf{H}_{\boldsymbol{m}}$ \\
Belo Horizonte & 2.109 & $24,1 \%$ & 2.491 .109 & 0,8 \\
Caeté & 63 & $100 \%$ & 43.395 & 1,5 \\
Itaguara & 44 & $100 \%$ & 53.087 & 3,4 \\
Lagoa Santa & 74 & $69,2 \%$ & 29.873 & 1,3 \\
Mateus Leme & 60 & $100 \%$ & 36.382 & 2,0 \\
Matozinhos & 50 & $100 \%$ & 88.672 & 1,4 \\
Nova Lima & 95 & $43,3 \%$ & 62.473 & 1,1 \\
Pedro Leopoldo & 45 & $71,4 \%$ & 133.528 & 0,7 \\
Sabará & 55 & $46,6 \%$ & 214.830 & 0,4 \\
Santa Luzia & 75 & $100 \%$ & 116.506 & 0,3 \\
Vespasiano & 71 & $100 \%$ & 0,6 \\
\hline
\end{tabular}

\section{CONSIDERAÇÕES FINAIS}

Neste estudo, as características dos hospitais filantrópicos da RMBH foram comparadas com aquelas levantadas no âmbito nacional por Barbosa et al. $^{3}$ e Portela et al. ${ }^{5}$. Percebeu-se que há uma grande semelhança entre os dois recortes. Em ambos houve predominância de hospitais gerais sobre os hospitais especializados. Os gerais tendem a estar distribuídos pelos municípios menores, enquanto os hospitais especializados, em sua maioria, concentram-se nas capitais. Na RMBH, em todos os municípios menores onde há hospitais filantrópicos, esse número é limitado a uma unidade, tratando-se, na maioria dos casos, do único hospital do município, fato que corrobora a importância dessas organizações para a saúde local.

Também foi mostrado que o número de Leitos em Hospitais Filantrópicos por Mil Habitantes $\left(L_{H F} /{ }_{M} H\right)$ cresceu na $\mathrm{RMBH}$, bem como na capital e no conjunto dos municípios menores. Esse resultado contrastou com a tendência de queda encontrada nos dados do IBGE referentes ao indicador que considera leitos de hospitais públicos, privados e filantrópicos. O indicador $L_{H F} /{ }_{M} H$ do grupo dos municípios menores foi muito próximo daquele da capital Belo Horizonte.

No âmbito da RMBH, Itaguara foi o único município, dentre aqueles que dispõem de apenas uma unidade hospitalar, sendo ela filantrópica, que apresentou o $\mathrm{L} /{ }_{M} \mathrm{H}$ maior que o mínimo recomendado pela OMS. Como poucas pesquisas sobre capacidade assistencial hospitalar foram realizadas no âmbito regional, deixa-se como indicação para futuros estudos calcular o indicador Leitos por Mil Habitantes para diversas regiões do país e compará-lo com o mínimo recomendado pela OMS.

\section{REFERÊNCIAS}

1. Brasil. Ministério da Saúde. Secretaria Nacional de Ações Básicas de Saúde. Coordenação de Assistência Médica e Hospitalar. Conceitos e definições em saúde. Brasília: Ministério da Saúde; 1977. 39 p.

2. Portela MC, Lima SML, Vasconsellos MM, Barbosa PR, Ugá MAD. Estudo sobre hospitais filantrópicos no Brasil. Rev Adm Pública. 2000;34(2):1-11.

3. Barbosa PR, Portela MC, Ugá MAD, Vasconsellos MM, Gerschman SV, Lima SML. Hospitais filantrópicos no Brasil. Rio de Janeiro: BNDES Social; 2002. 195 p

4. Lima SML, Barbosa PR, Portela MC, Ugá MAD, Vasconcellos MM, Gerschman S. Caracterização gerencial dos hospitais filantrópicos no Brasil. Cad Saude Publica. 2004;20(5):1249-61. http://dx.doi.org/10.1590/ S0102-311X2004000500019. PMid:15486668.

5. Portela MC, Lima SML, Barbosa PR, Vasconsellos MM, Ugá MAD, Gerschman S. Caracterização assistencial de hospitais filantrópicos no Brasil. Rev Saude Publica. 2004;38(6):811-8. http://dx.doi.org/10.1590/ S0034-89102004000600009. PMid:15608899.

6. Instituto Brasileiro de Geografia e Estatística. Pesquisa Assistência MédicoSanitária (AMS) [Internet]. Rio de Janeiro; 2009 [citado em 2017 mar 23]. Disponível em http://seriesestatisticas.ibge.gov.br/series.aspx?vcodigo=MS33
7. Gentili M, Isett K, Serban N, Swann J. Small-area estimation of spatial access to care and its implications for policy. J Urban Health. 2015;92(5):864-909. http://dx.doi.org/10.1007/s11524-015-9972-1. PMid:26282563.

8. Lima SML, Portela MC, Ugá MAD, Barbosa PR, Gerschman S, Vasconcellos MM. Hospitais filantrópicos e a operação de planos de saúde próprios no Brasil. Rev Saude Publica. 2007;41(1):116-23. http://dx.doi.org/10.1590/ S0034-89102007000100016. PMid:17273642.

9. Hart LG, Amundison BA, Rosenblatt RA. Is there a role for the small rural hospital? J Rural Health. 1990;6(2):101-18. http://dx.doi. org/10.1111/j.1748-0361.1990.tb00194.x. PMid:10170543.

10. Rede Interagencial de Informações para a Saúde. Indicadores básicos para a saúde no Brasil: conceitos e aplicações. 2. ed. Brasília: Organização PanAmericana da Saúde; 2008. 350 p.

11. Rodrigues J Fo. A distribuição dos recursos de saúde no Brasil: a administração da desigualdade. Rev Adm Empres. 1987;27(3):52-7. http:// dx.doi.org/10.1590/S0034-75901987000300007.

12. Wennberg J, Gittelsohn A. Small area variations in health care delivery. Science. 1973;182(4117):1102-8. http://dx.doi.org/10.1126/science.182.4117.1102. PMid:4750608. 
13. Wennberg J, Gittelsohn A. Variations in medical care among small areas. Sci Am. 1982;246(4):100-11. http://dx.doi.org/10.1038/scientificamerican0482-120. PMid:7079718.

14. Castro MSM, Travassos C, Carvalho MS. Efeito da oferta de serviços de saúde no uso de internações hospitalares no Brasil. Rev Saude Publica. 2005;39(2):277-84. http://dx.doi.org/10.1590/S0034-89102005000200020. PMid:15895149.

15. Departamento de Informática do Sistema Único de Saúde. Indicadores de recursos [Internet]. Brasília: DATASUS; 2017 [citado em 2017 mar 23]. Disponível em: http://tabnet.datasus.gov.br/cgi/idb2000/fqe02.htm

16. Luiz OC, Heimann LS, Boaretto RC, Pacheco AG, Pessoto UC, Ibanhes LC, et al. Diferenças intermunicipais de condições de vida e saúde: construção de um indicador composto. Rev Saude Publica. 2009;43(1):115-22. http:// dx.doi.org/10.1590/S0034-89102009000100015. PMid:19169583.

17. Brasil. Ministério da Saúde. Nota técnica informativa [Internet]. Brasília: Ministério da Saúde; 2014 [citado em 2017 mar 23]. Disponível em: http:// tabnet.datasus.gov.br/cgi/idb2012/Nota_tecnica_informativa_reducao_leitos. pdf

18. Anderson GF, Reinhardt UE, Hussey PS, Petrosyan V. It's the prices, stupid: why the United States is so different from other countries. Health Aff. 2003;22(3):89-105. http://dx.doi.org/10.1377/hlthaff.22.3.89. PMid:12757275.

Recebido em: Jun. 14, 2017

Aprovado em: Dez. 14, 2018 